that he was obliged to betake himself into this hospital for relief.

Condition on admission.-The scrotum was about the size of a child's hesd, and the penis was imbedded in it. The right side of the scrotum was more swollen and prominent than the left. It was also hot to the feel, soft and yielding on pressure. On examining the swelling next morning after admission, distinct fluctuation was found both outside and inside the tunica raginalis.

On making an opening at the most depending part of this swelling about 3 pints of sanguineous pus with a few masses of broken down blood clot were let out; on exploring, the finger passed through a ragged opening in the sac within the tunica, and the testicle could be distinctly felt behind it. The cavities were then thoroughly washed out with carbolic acid lotion; a drainage tube was inserted, and the wound dressed antiseptically. There was a little febrile re-action after the operation, and the temperature ranged between $99^{\circ} \mathrm{F}$. to $101^{\circ} \mathrm{F}$, the discharge becoming dally less in quantity; but the sinus showed no tendency to contract, owing to the extremely thickened walls of the tunica. It was therefore resolved to remove them. Accordingly, on the 17th of February, one month and seren days after admission, the sinus was laid open throughout after the patient had been put under chloroform. The tunica raginalis was found to be enormously thickened and degenerated-fibro-cartilaginous, and lined by a rough fibrinous material; the parietal portion of it was en. tirely dissected off. There was very little bleeding from the wound; about a dozen ligatures were applied. The wound was then washed out, plugged with lint and carbolic oil, and kept open in case of oozing from the extensive cut surface. In the evening the dressings were taken out, a few bleeding points tied, and the wound was stitched up by double iron wire sutures. There was a little constitutional disturbance after this operation, and a little discharge began to issue from the upper and lower corners of the wound; when a drainage tube was passed through the lower opening, and then the discharge diminished in quantity. The ferer gradually abated. On the erening of 1st March he got an attack of fever with chilliness, and the discharge became fotid and increased from the lower sinus. On the next day the fever subsided, but the scrotum became painful; on the 3rd of March, the right side of the scrotum was swollen and fluctuating to the feel; a counter opening was made, and two drainage tubes were passed through from this new opening to the two old sinuses, at each corner of the wound. From that day the patient has experienced very little pain in the scrotum, which has gradually decreased in size. The discharge, however, continued foetid, though it became less in quantity. On the 9 th of March another small collection of pus was noticed on the posterior and outer part of the scrotum, a counter opening was made, and a drainage tube inserted. The discharge gradually grew healthier and scantier. The drainage tubes were taken out one after another; the swelling of the scrotum rapidly subsiding and the sinuses contracting: the right side of the scrotum has now become less than the left; the corerings of the testicle having contracted round and adhered to the surface of the gland. The spermatic cord, which was much enlarged and indurated, has also regained its normal condition.

REMarks. - This was a case of abscess of the tunica raginalis and scrotum consequent on hæmatocele, which appears to have supervened on a hydrocele of long standing. The interest of the case centres in the manner in which the very much thickened and degenerated tunica was treated. Authorities differ as to what ought to be done in these circumstances. Bryant advises such cases to be left to nature, but the recovery must be very tedious, and a very considerable swelling must remain; Syme adrises partial excision of the tunica, and Erichsen castration. The treatment adopted in this case has proved a perfect success, and is, I think, the correct plan in such cases.

\section{A MEDICO-LEGAL CASE.}

\section{By Charles Moore Jessop, Surgeon-Major, 4th Hussars.}

Tre following case occurred at Mhow in September 1865. Apart from its interest in a medical point of riew, it also possesses considerable interest legnlly in the determination of how far an external bruise, produced by a fall or blow, would cause the extensive apoplectic clot found after death. For it is to be noted that the deceased was seen alive in company with three European soldiers, one her husbaud and two her paramours, who were afterwards seen by several natives to carry and deposit her under a tree near her residence in the bazan, and then leave : and as death ultimately resulted, was it a case of murder or homicide? The hour was watch-setting -8 or 8.30 P.M.

Mrs. T., aged 28, was the wife of Private T. in the 6th Dragoons, and noted for her drunken and dissolute habits, possessing considerable personal attractions, and a stout healthylooking woman. Had been in hospital in June for pain in her right side, and again in July for diarrhœe. Her last admission was on the 5th September, at 11 P.M., in a state of insensibility, with her left cheek and temple bruised and swollen. The Apothecary, Mr. Hudson, gave her an emetic; the ejected matter smelt strongly of spirit. In two hours' time, after other remedial measures, there was slightly returning conscious. ness.

On the following morning it was noticed that she was paralysed on the right side; the right arm was perfectly motionless; the leg was just sensible to stimulus, and no more; there was eversion of the right eyeball. Pupils of both eyes contracted. Stertorous breathing was setting in ; the pulse was slow and feeble. Enema, cupping, sinapisms, with calomel and croton oil; this latter brought away a large black evacuation. The breathing hecame quiet, and the pulse less laboured.

On the 7th seemed improved; more motion in the right leg; eyeball less everted; swelling of face had subsided, and in an inarticulate manner she asked for the bed-pan; right side of face was paralysed, and she could not close the eyelia.

On the 8th took fluids readily; complained of being sore all over; was very low and feeble; pulse began to flag.

On the 9 th not so well; great restlessness; constant clench. ing of the left hand; right leg and arm quite motionless.

On the 10th there was trismus and insensibility. Eversion of the left eyeball; the right was now natural; a great deal of rigidity about the left arm with muscular twitchings. Her evacuations were passed involuntarily. Blisters to nape of neck and vertex remored the trismus, and she rallied somewhat.

On the morning of the 11th at $8 \mathrm{~A}$.r. stertorous breathing set in. The left eyeball became natural, and the right again was everted. Both eyelids were now closed. She continued in this state till 5.45 P.M., when sise died.

Autopsy 14 hours after death.

General aspect of this body was that, apparently, of a person at once healthy and plump, of ruddy complexion and dark hair. Cadaveric rigidity well marked. Post-mortem ecchymoses extensive and occupying all the dependent parts.

There is no appearance of blows or bruises on the head. On the face there is a large bruise over the left eye and jaw. The upper portions of both arms are very much bruised, also the left hand and lower part of the same arm. There are several bruises about the shoulders and left breast. Both legs also have several bruises on them. All these bruises are of recent origin or within a period of 8 days.

Head and Neck.-The scalp was deroid of blood, except posteriorly. The skull-cap was adherent in the neighbourhood of the longitudinal sinus. The dura mater had a dull aspect and marked green tinge over the right hemisphere, and it felt boggy as if fluid was beneath. There was a similar appearance, but not so noticeable over the left hemisphere. On raising the dura mater a large clot of blood was seen covering the whole of the right hemisphere and extending down the side of the skull to the tentorium cerebelli. There was very great compression of the brain from this clot, which appeared to be from half to three quarters of an inch in thickness, and in weight about six ounces. The veins of the pia mater were loaded with black blood; over the left hemisphere was a thin coagulated and bloody layer of serum. Opposite the left orbit was a small extravasation; the veins of the pia mater on this side did not present the black and turgid appearance of those on the opposite side. In the course of the longitudinal sinus and falx cerebri were marks of old adhesions; there was no effusion of blood at the base of the brain; about one ounce of cerebro-spinal fluid; the substance of the brain was firm; on section with numerous dark-red blood spots; the ganglin on each side looked paler than usual (perhaps from contrast).

There were no clots in the substance of the brain; no fluid in the ventricles; the arachnoid on the superior surface of the cerebellum was milky.

Larynx and trachea were deeply congested and of a purple tint; at the bifurcation of the trachea there was great congestion, particularly on the left side. 
Cavity of the thorax.-Pleurr healthy; lungs both fully congested, left more than the right; heart, much external fat, flabby, and small; its cavities were all empty; valves perfect; no pericardial fluid.

Cavity of the Abdomen. - There was here a large amount of adipose tissue, in the omenta, peritoneum, and around all the organs. Liver was adherent to the diaphragm by its left lobe and posterior portion of the right lobe. The whole organ was smaller than natural; the left lobe was atrophied, except a small portion, into a mass of cellular tissue. Glisson's capsule was portion, into a mass of cellular was nodulated, blood freely adherent to the right lobe, which was nodulated, blood this lobe exuding from large and small cut-vessels. Throughout this lobe were pea-sized points of cheesy matter beginning to soften. Todine gave a brown stain.

Spleen double the natural size. Substance firm, with adherent capsule.

Kidneys.-Cortical portion reduced. The organs were soft and full of blood, partaking of the plethoric condition of the whole system. Capsule not adherent.

The remining viscera were not examined, but it was observed that the veins of the omenta and mesenteries were all loaded with blood.

REMARKS.- -Were the "pea-sized points of cheesy matter beginning to soften" in existence before the apoplectic clot, or the rupture? As the large intestine unfortunately was not examined, it is As the large intestine unis subject, but the chances are, they impossible to ef innervation.

Did the external bruises accelerate death, and were they the result of accident or design?

At the Court of Inquest the statements of the three Europeans At the native evidence. The men well understood the gravity of their position, and, The men well understood man framed a story that would shield him.

The bruises about the body could hardly be held accountable for the lesion disclosed, but the large bruise on the left able for the lesion as a fist might have caused, with the absence of any direct injury to the head, might have produced the apoplexy by contre.coup.

It wns ghewn in evidence that, throughout the day of the 5th September, the woman had been filled with spirituous liquor, but as this was of common occurrence, it could not be sup. posed that apoplexy should have resulted without some other posed that apoplexy should sa that a violent altercation took assistant cause. It was shown that a violent altercation took place between two of the men and the woman, who was much excited, and her face. We may, therefore, assume that the left side of her face. plethoric condition of the somehow on the left cheel and eje, ment, and a berial rupture.

Presuming the the medical part of the case, still there remains the legal. Was the bruise the result of accident or design? Positively this the butecidental.

The following letter, which I, as a member in those days of The Court, addressed to the President, may help to answer the question.

"On reviewing in my own mind all the evidence we have been listening to for the last two days, I have come to the following conclusion :-

"From the evidence Private G. acknowledges to have been knocked down by Prirate T. Bombardier D. saw these two men knocked and Mrs. T. on the ground; he also assisted in lift. struggling and Mrs. $\mathrm{T}$. on the ground he interfered between Private ing Mrs. T. up. Private G. says he interled this and states he T. and his wife. Private T. acknowledges M. T. resented his pushed him away; Private G. also stas no external visible mark of interference. Now Private G. has sudden manner of learing the blow he describes, and his very sud if Private $T$., who is the couple is not satisfactory. Butso a little drunk, in re. described as being very angry and aimed a blow that should senting Private G.'s interference aime fell on Mrs. T.'s left have knocked him down, but accidentally whole problem; for cheek and eye, we have a solution to thence, yet at the same Mrs. T'. resented Private G.'s interference, time was anxious to protect him from her they had produced

"Privates $G$. and $T$, seeing the mischief they hab produced with Bombadier D.'s assistance, carried the insensible woman along the road, as described by the native evidence, underneath came to the place where she was thitimater hoping that she might recover; but each, being afraid of the consequences of being seen with one in her state, invented a separate story to account for $h$ is departure.

"Of course I beliere that the trinkets were taken by T. or G. to prevent robbery, and to be restored when she should come round. All the conflicting evidence may be explained in a similar way, and I beg, therefore, to suggest that Private T. and G. be searched for the missing property. $* * * * * *$

"I trust you will attach this letter to our proceeding, should you deem it of sufficient merit."

There was no testimony of intended murder, and the evidence considered, as in the above letter, reduces the case to accidental homicide. After the inquest Private T. was placed in the guard-room, and the missing trinkets were found in his box. He remained in confinement for 45 days, and was then relensed under instructions from head-quarters.

RAWULPINDEE, 27th March 1875.

\section{CASES FROM PRACTICE.}

By J. Slane, L.R.C.P., Civil Medical Officer, Gowalpara.

I send the following notes of two cases which have recently occurred in the Gowalpara jail, and which possess some interest pathologically and therapentically.

CASE I.-SANGUINEOUS APOPLEXY.

Chandmoni, female, aged 30 , had suffered recently from ague, which was followed by œdematous swelling of the face ague, which was forties. The œdema was slow in subsiding, and lower extremities. but illness at the weekly inspection of the prisoners, and she then made no complaint. I was informed that she had eaten heartily of the evening meal, and played for some time with the little child, for purchasing which she was then in jail. Soon after she fell asleep, and about 10 o'clock without any premonitory symptoms she was attacked by convulsions of an epileptiform character, and up till 12 o'clocis had six fits. In the tirst paroxysm I witnessed a low sobbing was uttered, the face paroxysm I withe right side, the mouth pursed up, and all was acial muscles were in violent spasmodic movement; the trunk and limbs were rigid and moved in violent tetanic-like spasms, but the trunk did not writhe or shift its position as spasms, broth issued from the mouth, and the tongue was in epilepsy Froth isom the absence of incisor teeth was not protruded; but from the dilated, and the conjunctivæ congested. bitten; the pupils were dilated, and about three minutes, the After the paroxysm, which laste 98 and weak. There was temperature was found 102 ; pulse 98 and weak. There was hyperæsthesia of the surface, and sprinkling with cold water appeared to induce the paroxysms. During the int

them she was comatose and breathing stertorously.

She had calomel ten grains, croton oil two drops, a turpenA sulphate of zinc emetic had been administered, but vomiting A sulphate of zinc een induced.

Rum $z_{i}$ in water $̧$ iv was given every hour by enema.

October 20 th - Comatose, and breathing stertorously. Temperature $101^{\circ} 70^{\prime}$ clock $\Lambda$. M., pulse 108 ; bowels have been freely moved, and has had but two fits since 2 o'clock A. M. Has made water twice, but none of it could be obtained; sho swallows now pretty well.
Bo Chloral hydrate ...

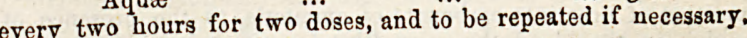
Rum and chicken broth enemata.

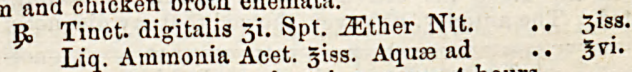
An ounce to be taken every 4 hours.

3 o'clock p.m.-Has had no fit; respiration is now free from $3 o^{\prime}$ clock p.m.- Has had no fit ; respiratin pinched; the bowels stertor, and she evinces signs of paince last report, the evacuations have been moved three times since last report, the evacuations being horribly offensive, and an is passed under her ; temapparently from the body.

perature $102^{\circ}$; pulse 114 , weak. Continue medicine.

October 21st. - Shews more per continues; pupils somewhat equal in both sides, but the stupor and frequent, but free from contracted; respirations shallow and frequent, but free from stertor; the respirato dull ; bowels twice moved, and has made and percussion note dhat milky in appearance; temperature $101.6^{\circ}$; pulse 114 ; urine sp. gr. 1015 , neutral, highly albuminous, about one-fifth.

Add ten grains of chloral hydrate to each dose of diuretic mixture. Turpentine stupes to chest; cupping and stupes to loins. 